\title{
Cyclodialysis Cleft Treatment Using a Minimally Invasive Technique
}

\author{
João Pinheiro-Costa ${ }^{a, b}$ António Benevides Melo ${ }^{a}$ \\ Ângela Maria Carneiro ${ }^{a, c}$ Fernando Falcão-Reis ${ }^{a, c}$ \\ ${ }^{a}$ Department of Ophthalmology, Hospital de São João, and Departments of ${ }^{\mathrm{b}}$ Anatomy and \\ 'Sense Organs, Faculty of Medicine, University of Porto, Porto, Portugal
}

\section{Key Words}

Cyclodialysis cleft · Hypotony · Cyclocryotherapy · Gas endotamponade

\begin{abstract}
Purpose: To report a case of a cyclodialysis cleft that was successfully managed with gas endotamponade and cyclocryotherapy. Methods: A 37-year-old male victim of a severe blunt ocular trauma was referred to our service for evaluation and treatment of a left eye hypotony. Clinical examination revealed an intraocular pressure of $2 \mathrm{~mm} \mathrm{Hg}$, a cyclodialysis cleft extending from the 11 to 1 o'clock positions and a hypotonic maculopathy. Left eye best corrected visual acuity (BCVA) was $3 / 10$. The patient failed to respond to conservative treatment with atropine $1 \%$, so a single bubble of $16 \%$ C2F 6 was injected into the vitreous cavity, followed by superior quadrant transconjunctival cyclocryotherapy. Results: After gas absorption, the intraocular pressure increased to $11 \mathrm{~mm} \mathrm{Hg}$ and became steady during the 24 months of follow-up. His hypotonic maculopathy resolved, and the BCVA improved to 9/10. Complete closure of the cyclodialysis cleft was documented with ultrasound biomicroscopy. Conclusion: Cryotherapy associated with gas endotamponade is a minimally invasive technique that could be considered for patients with cyclodialysis clefts that fail to respond to medical therapy.

(c) 2015 S. Karger AG, Basel
\end{abstract}

\section{Introduction}

A cyclodialysis cleft results from a disinsertion of the meridional ciliary muscle fibers from the scleral spur $[1,2]$. This creates an abnormal route of drainage of aqueous humor into the suprachoroidal space, resulting in chronic ocular hypotony $[1,2]$. Choroidal effusion, retinal folds, optic nerve edema, engorgement and stasis of retinal veins, shallow anterior

\section{KARGER 125}

João Pinheiro-Costa

Department of Ophthalmology, Hospital de São João

Faculty of Medicine, University of Porto

Al. Prof. Hernâni Monteiro, PT-4200-319 Porto (Portugal)

E-Mail joaopinh@ hotmail.com 
Pinheiro-Costa et al.: Cyclodialysis Cleft Treatment Using a Minimally Invasive Technique

chamber and cataract are frequent secondary complications of chronic ocular hypotony [1, 2]. Cyclodialysis clefts usually occur either as a surgical complication or after blunt ocular trauma $[1,3]$. The main goal of the treatment is to restore the apposition of the ciliary body to the sclera and consequently increase intraocular pressure (IOP). We report a case of a cyclodialysis cleft that was successfully managed with gas endotamponade and cyclocryotherapy.

\section{Case Report}

A 37-year-old male victim of a car crash with severe head injury and blunt ocular trauma was referred to our service for evaluation and treatment of a left eye hypotony 5 weeks after the accident. On ophthalmic examination, his best corrected visual acuity (BCVA) was 10/10 OD and 3/10 OS. Anterior segment examination revealed a shallow anterior chamber in the left eye and anisocoria, with a dilated left pupil. The IOP was $12 \mathrm{~mm} \mathrm{Hg}$ OD and $2 \mathrm{~mm}$ Hg OS. Left eye gonioscopy revealed a cyclodialysis cleft extending from the 11 to the 1 o'clock position, which was confirmed by ultrasound biomicroscopy (UBM; fig. 1). Dilated fundus examination revealed an optic disc edema and hypotonic maculopathy in the left eye (fig. 2). Right eye ophthalmic examination was unremarkable.

The patient started conservative treatment with topical atropine $1 \%$ twice daily, but after 4 weeks, there was no visual acuity improvement, and the left eye remained hypotonic. It was then decided to perform gas endotamponade followed by cyclocryotherapy. In the operating room and under general anesthesia, a single bubble of $16 \%$ hexafluoroethane-air mixture (C2F6, $0.5 \mathrm{ml}$ ) was injected into the vitreous cavity via the pars plana, followed by ocular decompression through a paracentesis of the anterior chamber. Superior quadrant transconjunctival cryotherapy was then performed (8 applications, each with a duration of $20 \mathrm{~s}$ and a temperature of $-80^{\circ} \mathrm{C}, 2 \mathrm{~mm}$ behind the limbus). The patient was told to rest with his head up for 1 week to achieve endotamponade. Atropine $1 \%$ twice daily was maintained for 2 months.

During the first week after the procedure, the IOP increased to $11 \mathrm{~mm} \mathrm{Hg}$. There was no hypertensive spike after cleft closure or intraocular inflammation in the postoperative period. Following gas absorption, at the third week, the IOP was $12 \mathrm{~mm} \mathrm{Hg}$. One month after the procedure, there was a complete resolution of the hypotonic maculopathy, and the BCVA improved to 9/10. Complete closure of the cleft and ciliary body reattachment was confirmed with gonioscopy and UBM (fig. 3). The IOP became steady during the 24 months of follow-up.

Informed consent was obtained, and the study was conducted in accordance with the tenets of the Declaration of Helsinki.

\section{Discussion}

The management of cyclodialysis clefts requires a careful approach. The identification of the full extent and location of the cleft is of particular importance for appropriate treatment $[1,4]$. Initial management should be conservative since some patients recover spontaneously or with the use of mydriatics [2]. Topical atropine can help close the cleft through relaxation of the ciliary muscle, allowing the apposition of the detached meridional ciliary muscle to the sclera [2]. Corticosteroids should be avoided to facilitate inflammatory adhesion at the cleft site [1]. Various techniques have been described as an alternative to traditional direct 
Pinheiro-Costa et al.: Cyclodialysis Cleft Treatment Using a Minimally Invasive Technique

cyclopexy; however, a review of the published literature does not indicate a clear gold standard technique $[1,3,5,6]$.

If medical treatment fails, further management with argon laser photocoagulation, applied within the cleft, seems to be a good option in small clefts, but an adequate gonioscopy is a problem in patients with a too shallow anterior chamber [2,7]. The laser can induce an inflammatory response, which triggers cleft closure. Other techniques like internal cerclage with a capsular tension ring or the haptics of large IOLs to compress the cleft against the sclera and achieve closure have recently been described $[3,8,9]$. Nevertheless, the number of studies evaluating their efficacy and safety is still scarce.

The use of transconjunctival cryotherapy, isolated or in conjunction with pars plana vitrectomy and internal gas endotamponade, has been applied in the treatment of persistent cyclodialysis clefts $[5,10]$. Helbig and Foerster [10] described the successful management of hypotonous cyclodialysis with pars plana vitrectomy, gas tamponade and cryotherapy. However, this technique requires an experienced vitreoretinal surgeon and has the risks connected with vitrectomy.

Traditional direct cyclopexy involves the creation of a partial or full-thickness scleral flap and suture of the ciliary body directly to the sclera. Direct cyclopexy is often a definitive procedure in patients where conservative management has failed. Nevertheless, this is an invasive technique with the risk of major hemorrhage from the ciliary body or endophthalmitis $[1,2]$.

Ceruti et al. [5] reported the successful resolution of a large cleft using gas endotamponade with a single bubble of $20 \%$ sulfur hexafluoride (SF6) in conjunction with cryotherapy. This technique combines the principle of direct mechanical apposition of the detached ciliary muscle to the sclera by a gas bubble and inflammation and scar induction by cryotherapy [5]. In our case, a similar technique was used, although with a different type of gas. We injected C2F6 instead of SF6 as the former takes longer to absorb and therefore has a longer-lasting effect; our anatomic and visual results were also good.

Cryotherapy associated with gas endotamponade is a rapid and technically simple surgical procedure that can be performed on an outpatient basis and without the need of direct visualization of the cyclodialysis cleft. In spite of being an intraocular procedure, this technique is minimally invasive and does not require an experienced surgeon. Although to the best of our knowledge this is only the second published case to be treated with this technique, we believe that this procedure should be used in the treatment of cases that fail to respond to medical therapy before other more invasive techniques are tried.

\section{Acknowledgements}

We thank José Silva Cotta, MD, and João Paulo Macedo, MD, for the help in the follow-up of this case.

\section{Disclosure Statement}

The authors have no conflicts of interest or financial support to declare. 
Case Reports in

Ophthalmology

\begin{tabular}{l|l}
\hline \multicolumn{2}{l|}{ Case Rep Ophthalmol 2015;6:66-70 } \\
\hline DOI: $10.1159 / 000375442$ & $\begin{array}{l}\text { C } 2015 \text { S. Karger AG, Basel } \\
\text { www.karger.com/cop }\end{array}$ \\
\hline
\end{tabular}

Pinheiro-Costa et al.: Cyclodialysis Cleft Treatment Using a Minimally Invasive Technique

\section{References}

$\checkmark 1$ Ioannidis AS, Barton K: Cyclodialysis cleft: causes and repair. Curr Opin Ophthalmol 2010;21:150-154.

-2 Aminlari A, Callahan CE: Medical, laser, and surgical management of inadvertent cyclodialysis cleft with hypotony. Arch Ophthalmol 2004;122:399-404.

-3 Malandrini A, Balestrazzi A, Martone G, Tosi GM, Caporossi A: Diagnosis and management of traumatic cyclodialysis cleft. J Cataract Refract Surg 2008;34:1213-1216.

-4 Trikha S, Turnbull A, Agrawal S, Amerasinghe N, Kirwan J: Management challenges arising from a traumatic 360 degree cyclodialysis cleft. Clin Ophthalmol 2012;6:257-260.

5 Ceruti P, Tosi R, Marchini G: Gas tamponade and cyclocryotherapy of a chronic cyclodialysis cleft. Br J Ophthalmol 2009;93:414-416.

-6 Ioannidis AS, Bunce C, Barton K: The evaluation and surgical management of cyclodialysis clefts that have failed to respond to conservative management. Br J Ophthalmol 2014;98:544-549.

7 Ormerod LD, Baerveldt G, Sunalp MA, Riekhof FT: Management of the hypotonous cyclodialysis cleft. Ophthalmology 1991;98:1384-1393.

-8 Yuen NS, Hui SP, Woo DC: New method of surgical repair for 360-degree cyclodialysis. J Cataract Refract Surg 2006;32:13-17.

-9 Mardelli PG: Closure of persistent cyclodialysis cleft using the haptics of the intraocular lens. Am J Ophthalmol 2006;142:676-678.

10 Helbig H, Foerster MH: Management of hypotonous cyclodialysis with pars plana vitrectomy, gas tamponade, and cryotherapy. Ophthalmic Surg Lasers 1996;27:188-191.

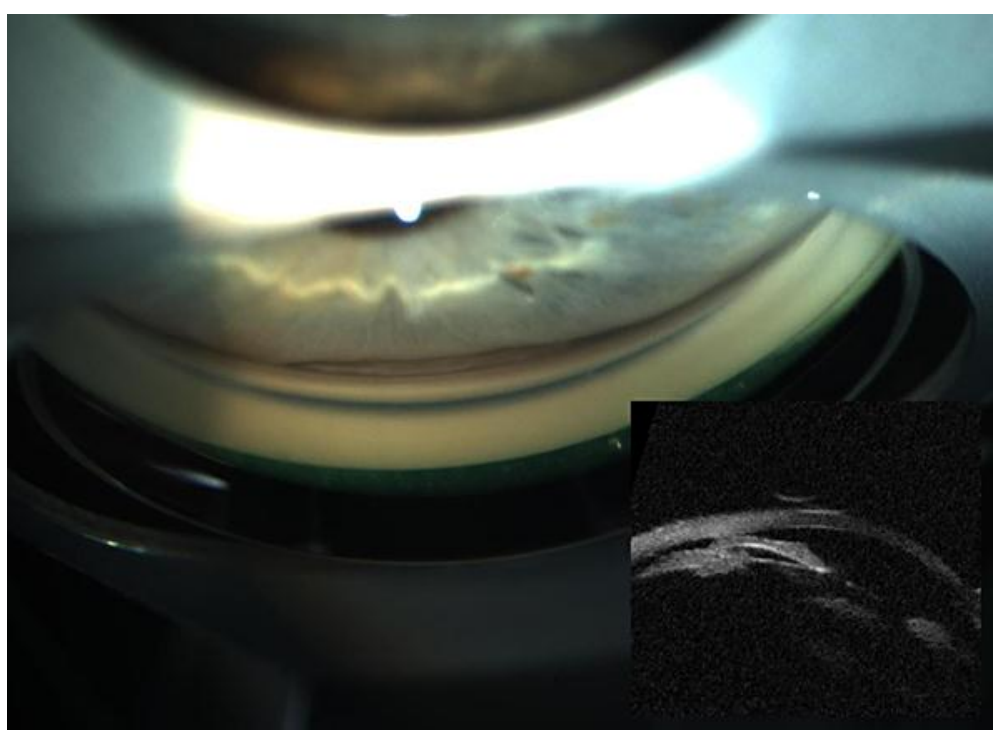

Fig. 1. Superior cyclodialysis cleft on gonioscopy (larger image). Disinsertion of the ciliary body from the scleral spur and ciliary body detachment on UBM (smaller image). 
Case Reports in

Ophthalmology

\begin{tabular}{l|l}
\hline Case Rep Ophthalmol 2015;6:66-70 \\
\hline DOI: $10.1159 / 000375442$ & $\begin{array}{l}\text { C 2015 S. Karger AG, Basel } \\
\text { www.karger.com/cop }\end{array}$ \\
\hline
\end{tabular}

Pinheiro-Costa et al.: Cyclodialysis Cleft Treatment Using a Minimally Invasive Technique

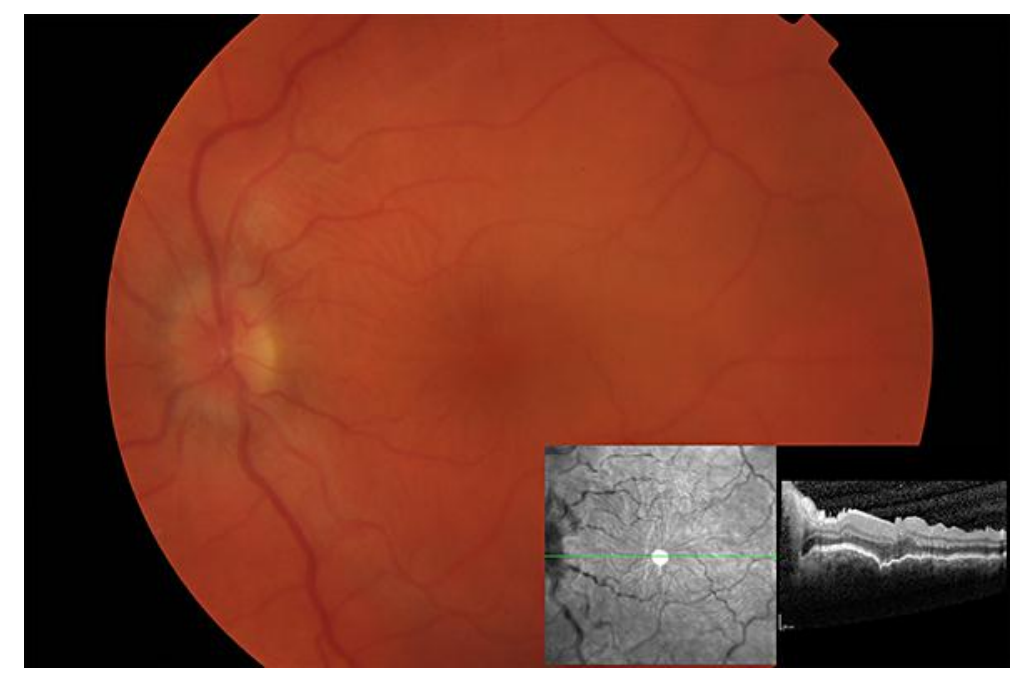

Fig. 2. Retinography and spectral domain optical coherence tomography showing hypotonic maculopathy.

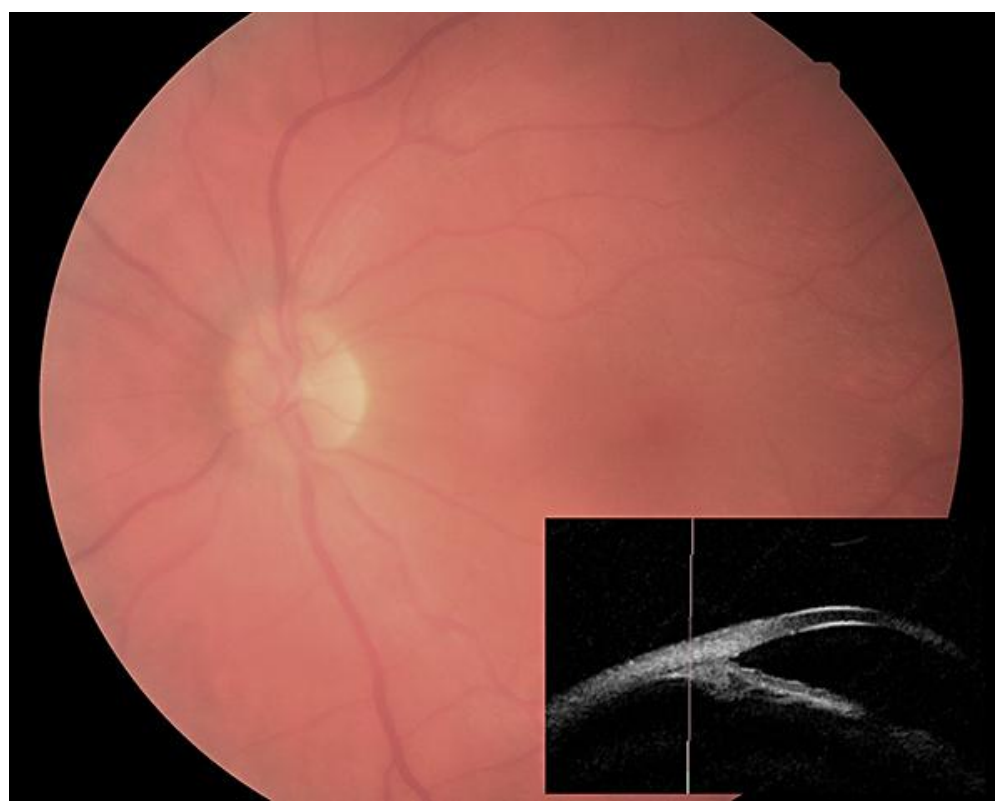

Fig. 3. Retinography and UBM at the end of follow-up. Note the disappearance of the hypotonic maculopathy and the reattachment of the ciliary body. 